\title{
The risk factors of diabetic nephropathy in Taiwan, including old age, hypertension and aspirin therapy
}

\author{
Yu-Wen Cheng • Usman Malik • Shih-Liang Chang
}

Received: 11 November 2012 / Accepted: 12 February 2013 /Published online: 21 May 2013

(C) Research Society for Study of Diabetes in India 2013

\section{Dear editor}

Taiwan has the highest prevalence and incidence of dialysis in the world and diabetes mellitus (DM) causes about $40 \%$ of end-stage renal failure [1]. Therefore, we would like to investigate the risk factors of diabetic nephropathy. A total of 379 Taiwanese treated for type 2 DM at least one year were analyzed from January 2010 to April 2011. Patients with hypertension (HTN) had lower estimated glomerular filtration rate (eGFR) than those without HTN $(64.7 \pm 18.3$ vs $70.9 \pm 20.2 \mathrm{ml} / \mathrm{min}, P<0.005)$, but those who accepted angiotensin-converting enzyme inhibitor (ACEI) or angiotensin II receptor blocker (ARB) therapy showed no harmful effects to renal function. Patients who took aspirin presented lower eGFR than those who didn't use aspirin $(60.4 \pm 19.8 \mathrm{vs}$ $67.7 \pm 19.5 \mathrm{ml} / \mathrm{min}, P<0.05)$. After one year of observation, patients with microalbuminuria had lower eGFR than those with normoalbuminuria (64.9 \pm 19.7 vs $69.3 \pm 19.8 \mathrm{ml} / \mathrm{min}, P<$ $0.05)$; in the original normoalbuminuria patients, those who developed microalbuminuria were older than those remained normoalbuminuria ( $68.3 \pm 12.4$ vs $62.7 \pm 11.5$ years, $P<0.05)$. Patients with poor blood glucose control ( $\mathrm{HbA} 1 \mathrm{c} \geqq 7.0 \%)$ had a greater chance of worsening renal parameters.

ADA suggested the use of low dose aspirin therapy as a primary prevention strategy for diabetic subjects at increased cardiovascular risk [2]. In the present sample

Y.-W. Cheng $\cdot$ U. Malik

Department of Internal Medicine, Lee's General Hospital,

Miaoli, Taiwan

\section{S.-L. Chang $(\bowtie)$}

Department of Medicinal Botanicals and Health Applications,

Da-Yeh University, No.168, University Rd., Dacun Changhua, Taiwan 51591

e-mail: slc0124@gmail.com it was reflected, showing that a single drug alone cannot make a difference and requires multifactorial intervention. ADA recommended ACEI or ARB as the treatment of choice for HTN in diabetic patients and the therapeutic goal of HbAlc was less than $7.0 \%$ because it was associated with the preservation of renal function [2, 3]. Rifkin et al. found that microalbuminuria was associated with an over $50 \%$ increase in mortality risk in the elderly. In cases where microalbuminuria combined with chronic kidney disease, there was a nearly 4-fold mortality risk [4].

In conclusion, aspirin therapy, older age and hypertension are the risk factors of diabetic nephropathy. Good blood glucose control could conserve normoalbuminuria.

Conflicts of interest None

\section{References}

1. USRDS. USRDS Annual Data Report, International comparison. Am J Kidney Dis. 2011;57 suppl 1:e383-96.

2. American Diabetes Association. Standards of medical care in diabetes-2011. Diabetes Care. 2011;34(supp 1):S11-61.

3. Raile K, Galler A, Hofer S, Herbst A, Dunstheimer D, Busch P, et al. Diabetic nephropathy in 27,805 children, adolescents, and adults with type 1 diabetes: effect of diabetes duration, A1C, hypertension, dyslipidemia, diabetes onset, and sex. Diabetes Care. 2007;30:2523-8.

4. Rifkin DE, Katz R, Chonchol M, Fried LF, Cao J, de Boer IH, et al. Albuminuria, impaired kidney function and cardiovascular outcomes or mortality in the elderly. Nephrol Dial Transplant. 2010;25:1560-7. 